\title{
AN UNORTHODOX PARAMETRIC MEASURE OF INFORMATION AND CORRESPONDING MEASURE OF INTUTIONISTIC FUZZY INFORMATION
}

\author{
${ }^{1} \mathrm{P}$. Jha, ${ }^{2}$ Vikas Kumar Mishra
}

${ }^{1}$ Department of Mathematics, Y.J. Govt. Chhattisgarh P.G. College, Raipur, Chhattisgarh, India

${ }^{2}$ Department of Mathematics, Rungta College of Engineering and Technology, Raipur, Chhattisgarh, India

Keywords: measure of entropy, directed divergence, intutionistic fuzzy entropy, intutionistic fuzzy directed divergence, information theory.

Abstract: A new parametric function

$$
v_{a}(p)=\log (1+a)-\sum_{i=1}^{n} \log \left(\frac{1+a p_{i}}{p_{i}}\right)-1, a>0
$$

is proposed for the probability distribution $p_{1}, p_{2}, p_{3}, \ldots \ldots \ldots p_{n}$ and its properties are studied. In this paper the given functions is twice differentiable and is used to obtain the related measure of directed divergence, measure of intutionistic fuzzy entropy, measure of intutionistic fuzzy directed divergence. We also investigate the monotonic character of the proposed function.

\section{Introduction}

In the present paper we draw our inspiration for obtaining a new parametric measure of entropy which is the joint effect of measures of information due to Kapur [6] and Burg [2]. In 1948, C.E. Shannon [8] gave the measure

$$
S(P)=-\sum_{i=1}^{n} p_{i} \log p_{i}
$$

to measure its uncertainty or entropy. It can also be regarded as a measure of equality of $p_{1}, p_{2}, p_{3}, \ldots \ldots \ldots p_{n}$ among themselves.

Later in 1972, J.P. Burg [2] and J.N. Kapur [6] gave the measure

$$
B(P)=\sum_{i=1}^{n} \log p_{i}
$$

and

$$
K_{p}(P)=-\sum_{i=1}^{n} p_{i} \log p_{i}+\frac{1}{a} \sum_{i=1}^{n}\left(1+a p_{i}\right)-\frac{1}{a}(1+a) \log (1+a)
$$

Shannon's and Burg's measures do not have any parameter, while Kapur's measure has one pararameter. When maximized by Lagrange's method, subject to linear constraints on probabilities, the measures due to Shannon, Burg and Kapur always give non-negative probabilities. Shannon's measure has been the most successful and most widely used measure. Burg's measure has also been successful, but it is always negative and as such it is hard to interpret it as a measure of uncertainty. However, it can be used for entropy maximization purposes and it has been so used. Moreover, its maximum value decreases with $\mathrm{n}$ and this is not a desirable property for a measure of entropy. In the present discussion, we modify the Burg's and Kapur's measures to obtain a new parametric measure of entropy. We shall also study the properties of the measure and also investigate the related directed divergence motivated by Kullback-Liebler [7]. In this paper we introduce the 
corresponding measure of intutionistic fuzzy entropy and measure of intutionistic fuzzy directed divergence.

Here we define intutionistic fuzzy set as given by Atanasov (1983) [1] and then we discuss about the conditions of measure of intutionistic fuzzy entropy.

Intutionistic fuzzy set: - Let a set be fixed. An intutionistic fuzzy set (IFs) $A$ of $E$ is an object having the form $A=\left\{\left\langle x, \mu_{A}(x), v_{a}(x)\right\rangle x \in E\right\}$ where the function $\mu_{A}: E \rightarrow[0,1]$ and $v_{a}: E \rightarrow[0.1]$ define respectively the degree of membership and degree of non membership of the element $x \in E$ to the set $A$, which is a subset of $E$ and for every $x \in E, 0 \leq \mu_{A}(x)+v_{a}(x) \leq 1$

\section{Conditions for measures of intutionistic fuzzy entropy:-}

1. It should be defined in the range $0 \leq \mu_{A}(x)+v_{a}(x) \leq 1$

2. It should be continuous in this range.

3. It should be zero when $\mu_{A}(x)=0$ and $v_{a}(x)=1$.

4. It should not be changed when $\mu_{A}(x)$ changed in to $v_{a}(x)$.

5. It should be increasing function of $\mu_{A}(x)$ in the range $0 \leq \mu_{A}(x) \leq 0.5$ and decreasing function of $v_{a}(x)$ in the range $0 \leq v_{A}(x) \leq 0.5$

6. It should be concave function $\mu_{A}(x)$.

\section{Our Results}

\subsection{Some Properties of the New Measures of Information}

The measure is defined by

$$
v_{a}(p)=\log (1+a)-\sum_{i=1}^{n} \log \left(\frac{1+a p_{i}}{p_{i}}\right)-1, a>0
$$

It has the following properties:

1. It is a continuous function of $p_{1}, p_{2}, p_{3}, \ldots \ldots p_{n}$ so that it changes by a small amount when $p_{1}, p_{2}, p_{3}, \ldots \ldots \ldots p_{n}$ change by small amounts.

2 . It is a permutationally symmetric function of $p_{1}, p_{2}, p_{3}, \ldots \ldots p_{n}$ i.e. the function does not change when $p_{1}, p_{2}, p_{3}, \ldots \ldots \ldots p_{n}$ are permuted among themselves.

3. It is maximum, subject to natural constrai

When $p_{1}=p_{2}=\cdots \ldots \ldots \ldots=p_{n}=\frac{1}{n}$

$$
\sum_{i=1}^{n} p_{i}=1
$$

4. The maximum value is an increasing function of $\mathrm{n}$. In fact the maximum value is given by

$$
\begin{aligned}
f(n) & =\log (1+a)-\sum_{i=1}^{n} \log (1+a / n)+\sum_{i=1}^{n} \log \frac{1}{n}-1 \\
& =\log (1+a)-n \log \left(\frac{n+a}{n}\right)+n \log \frac{1}{n}-1
\end{aligned}
$$

so that $f^{\prime}(n)=\frac{-n}{n+a}+\log \left(\frac{1}{n+a}\right)$

and $f^{\prime \prime}(n)=(-a) /(n+a)^{2}-1 /(n+a)<0$

So that $f(n)$ is a concave function of $n$ and $f^{\prime}(n)$ is a monotonic increasing function of $n$.

Now $\quad f^{\prime}(n)=\frac{-n}{n+a}+\log \left(\frac{1}{n+a}\right)$ 


$$
\begin{aligned}
& =\frac{a}{n+a}-1+\log \left(\frac{1}{n+a}\right) \\
& =\frac{a}{y}-1+\log \left(\frac{1}{1}\right) \\
& =\frac{a-y+\log \left(\frac{1}{y}\right)}{y} ; y=n+a>0
\end{aligned}
$$

Since

$$
y>0 \text { and } a-y+\log \left(\frac{1}{y}\right)<0 \text { when } y>0
$$

We get

$$
f^{\prime}(n)<0
$$

So that $f(n)$ is monotonically increasing.

Thus, $v_{a}(p)$ satisfies all the important properties satisfied by Shannon's measure of entropy except additivity and recursivity. However, these properties are unimportant for entropy maximization purpose and hence, $v_{a}(p)$ is an entropy.

\subsection{Corresponding Measure of Intutionistic Fuzzy Information}

$$
V(A)=\log (1+a)-\sum_{i=1}^{n} \log \left(\frac{1+a \mu_{A}\left(x_{i}\right)}{\mu_{A}\left(x_{i}\right)}\right)-\sum_{i=1}^{n}\left(\frac{1+a v_{A}\left(x_{i}\right)}{v_{A}\left(x_{i}\right)}\right)-1, a>0
$$

It has the following properties:

1. It is defined in the range $0 \leq \mu_{A}\left(x_{i}\right)+v_{A}\left(x_{i}\right) \leq 1$

2. It is continuous in this range

3. It is zero when $\mu_{A}\left(x_{i}\right)=0$ and $v_{A}\left(x_{i}\right)=1$

4. It does not changed when $\mu_{A}\left(x_{i}\right)$ changed in to $v_{A}\left(x_{i}\right)$

5. It is increasing function of $\mu_{A}\left(x_{i}\right)$ in the range $0 \leq \mu_{A}\left(x_{i}\right) \leq 0.5$ and decreasing function of $v_{A}\left(x_{i}\right) \quad$ in the range $0 \leq \mu_{A}\left(x_{i}\right) \leq 0.5$

Let $\mu_{A}\left(x_{i}\right)=s$ and $v_{A}\left(x_{t}\right)=t$ then

$$
f(s, t)=\log (1+a)-\log (1+a s)+\log s-\log (1+a t)+\log t-1
$$

differentiate wrt. $s$ we get

So It is a concave function of $\mu_{A}\left(x_{i}\right)$

$$
\begin{aligned}
f^{\prime}(s, t) & =\frac{-1}{1+a s}+\frac{1}{s} \\
f^{\prime}(s, t) & =\frac{a}{(1+a s)^{2}}-\frac{1}{s^{2}}<0
\end{aligned}
$$

Since all the conditions for measures of intutionistic fuzzy entropy are satisfying very well and therefore (2.2.1) is a valid measure of intutionistic fuzzy entropy.

\subsection{Corresponding Measure of Directed Divergence}

Motivated by Kullback and Liebler [8] we get the corresponding measure of directed divergence,

$$
D_{a}(P: Q)=\sum_{i=1}^{n} p_{i} \log \frac{p_{i}}{q_{i}}-\sum_{i=1}^{n} p_{i} \log \frac{1+a p_{i}}{1+a q_{i}}
$$

Here $D_{a}(P: Q)$ satisfies the properties $D_{a}(P: Q) \geq 0$, vanishes iff $Q=P$ and is a convex function of both $p_{1}, p_{2}, p_{3}, \ldots \ldots p_{n}$ and $q_{1}, q_{2}, q_{3}, \ldots \ldots q_{n}$ 


\subsection{Corresponding Measure of Intutionistic Fuzzy Directed Divergence}

$$
\begin{gathered}
D_{a}(A: B)=\sum_{i=1}^{n} \mu_{A}\left(x_{i}\right) \log \frac{\mu_{A}\left(x_{i}\right)}{\mu_{B}\left(x_{i}\right)}+\sum_{i=1}^{n} v_{A}\left(x_{i}\right) \log \frac{v_{A}\left(x_{i}\right)}{v_{B}\left(x_{i}\right)}-\sum_{i=1}^{n} \mu_{A}\left(x_{i}\right) \log \frac{1+a \mu_{A}\left(x_{i}\right)}{1+a \mu_{B}\left(x_{i}\right)} \\
-\sum_{i=1}^{n} v_{A}\left(x_{i}\right) \log \frac{1+a v_{A}\left(x_{i}\right)}{1+a v_{B}\left(x_{i}\right)}
\end{gathered}
$$

It is a convex function of $\mu_{A}\left(x_{i}\right)$. Since $D_{a}(A: B)$ satisfies all the conditions for measures of intutionistic fuzzy directed divergence therefore (2.4.1) is a valid measure of intutionistic fuzzy directed divergence.

\section{References}

[1] Atanassov K. intuitionistic fuzzy sets, VII ITKR's Session, Sofia, June 1983, Deposed in Central Sci.- Techn. Library of Bulg. Acad. of Sci., 1697/84).

[2] J.P. Burg, The relationship between maximum entropy spectra and maxi-mum likelihood spectra, In: Modern Spectra Analysis (Ed. D.G. Childers), MSA (1972), 130-131.

[3] J.N. Kapur, Four families of measures of entropy, Int. Jour. Pure and App. Maths., 17, No. 4, (1986),429-449.

[4] J.N. Kapur, Burg and Shannon's measure of entropy as limiting case of a family of measures of entropy, Proc. Nat. Acad. Sciences, 61A, Part III (1991), 371-387.

[5] J.N. Kapur, Measures of Fuzzy Information, Mathematical Sciences, Trust Society (1996).

[6] J.N. Kapur, Measures of Information and Their Application, Wiley Eastern, New Delhi (1997).

[7] S. Kullback, R.A. Liebler, On Information and Sufficiency, Ann. Math.Stat., 22 (1951), 77-89.

[8] C.E. Shannon, A mathematical theory of communication, Bell System Tech. J., 27 (1948), 379423, 623-659.

[9] C.M. Thomas, J.A. Thomas, Elements of Information Theory, Second Edition, John Wiles and Sons (2006). 